\title{
Raman study of the polybenzimidazole-phosphoric acid interactions in membranes for fuel cells
}

\author{
Fosca Conti, ${ }^{a b}$ Anne Majerus, ${ }^{a}$ Vito Di Noto, ${ }^{b}$ Carsten Korte, ${ }^{a}$ Werner Lehnert ${ }^{a}$ \\ and Detlef Stolten ${ }^{a}$
}

Received 22nd February 2012, Accepted 6th May 2012

DOI: $10.1039 / \mathrm{c} 2 \mathrm{cp} 40553 \mathrm{a}$

Poly(2,5-benzimidazole) (AB-PBI) membranes are investigated by studying the FT-Raman signals due to the benzimidazole ring vibration together with the $\mathrm{C}-\mathrm{C}$ and $\mathrm{C}-\mathrm{H}$ out-of- and in-plane ring deformations. By immersion in aqueous ortho-phosphoric acid for different time periods, membranes with various doping degrees, i.e. different molar fractions of acid, are prepared. The chemical-physical interactions between polymer and acid are studied through band shifting and intensity change of diagnostic peaks in the $500-2000 \mathrm{~cm}^{-1}$ spectral range. The formation of hydrogen bonding networks surrounding the polymer seems to be the main reason for the observed interactions. Only if the AB-PBI polymer is highly doped, the Raman spectra show an additional signal, which can be attributed to the presence of free phosphoric acid molecules in the polymer network. For low and intermediate doping degrees no evidence for free phosphoric acid molecules can be seen in the spectra. The extent of the polymer-phosphoric acid interactions in the doped membrane material is reinvestigated after a period of one month and the stability discussed. Our results provide insight into the role of phosphoric acid as a medium in the conductivity mechanism in polybenzimidazole.

\section{Introduction}

Increasing populations and growing industrialization raise the energy demand in the world. Recent international agreements have indicated one feasible solution to make the development compatible with the environment: the widespread adoption of technologies to reduce energy consumption and the environmental impact of human activities. In this context, fuel cells (FCs) play an important role among the energy conversion devices that constitute a real prospect in the medium and long term.

Polymer Electrolyte Membrane FCs (PEMFCs) based on Nafion or other perfluorosulfonated polymers are widely used, although there are two main limitations: the high cost and the necessity to fully hydrate the membranes to keep their high proton conductivity. ${ }^{1,2}$ This also limits the working temperature below ca. $100{ }^{\circ} \mathrm{C}$ at ambient pressure, since the membrane dehydrates and the conductivity decays sharply above this temperature. High working temperatures would benefit PEMFC performance because of a higher $\mathrm{CO}$ tolerance, faster electrode kinetics and the existence of residual heat useful for energy cogeneration. ${ }^{3,4}$

${ }^{a}$ Institute of Energy and Climate Research - Fuel Cells (IEK-3), Forschungszentrum Jülich GmbH, 52425 Jülich, Germany.

E-mail:f.conti@fz-juelich.de; Fax: +492461 616695;

Tel: + 492461619568

${ }^{b}$ Department of Chemical Sciences, University of Padova,

Via Marzolo 1, 35131 Padova, Italy.E-mail: fosca.conti@unipd.it;

Fax: + $390498275829 ;$ Tel: + 390498275118
In the last two decades, many non-fluorinated polymer materials for membranes have been considered as an alternative to Nafion. ${ }^{5}$ Acid-doped poly $\left(2,2^{\prime}\right.$-( $m$-phenylene) $-5,5^{\prime}$-bibenzimidazole $)$ (PBI) polymers have been studied as membranes for the use in High Temperature Polymer Electrolyte Fuel Cells (HT-PEFCs), since they can be used at temperatures as high as $200^{\circ} \mathrm{C}$ without humidification. ${ }^{6-10} \mathrm{PBI}$ is a polymer containing a basic functionality that allows the uptake of acid, which is responsible, and required, for the proton conduction. PBI based acid-base membranes have been proposed by several groups using heteroand homogeneous synthesis. ${ }^{911-20}$ The membranes show high conductivity at low humidity,$^{21}$ good thermal and mechanical strength, ${ }^{22}$ high $\mathrm{CO}$ tolerance ${ }^{23}$ and low gas crossover. ${ }^{24}$ Among the many possible $\mathrm{PBI}$ derivatives, a very promising material is poly(2,5-benzimidazole) (AB-PBI) ${ }^{25-27}$ It has been found that AB-PBI absorbs phosphoric acid (PA) more efficiently than PBI when treated with equal concentrated solutions. ${ }^{25,28-30}$ Furthermore, AB-PBI membranes have the same performances as PBI under the same conditions and can be produced easily and more safely from a single cheap monomer. ${ }^{31}$

The use of Raman spectroscopy as a tool to study PBI type polymers is very rare, although there are beneficial aspects: the method is rapid, nondestructive and does not require sample preparation. ${ }^{32}$ Recently, some PBI derivatives have been characterized by their Raman spectra through the attribution of many bending and stretching active modes. ${ }^{33}$ In a few cases the spectra have been utilized to establish the presence of 
interactions between the polymer and PA in doped membranes. The formation of hydrogen bonding can be observed mainly in band shifting and intensity change. ${ }^{34}$ Unfortunately, to the best of our knowledge, no complete series of measurements for different doping concentrations can be found in the literature.

In the present study, we report on a Fourier Transform Raman (FT-Raman) investigation of AB-PBI polymer membranes doped with various concentrations of ortho-phosphoric acid. Four differently doped membranes are examined and a correlation between the optical signal and the doping degree is analysed. The spectral features observed in the $500-2000 \mathrm{~cm}^{-1}$ wavenumber range are discussed. An attempt to assign the nature of the various bands is presented. Correspondences based on previous reports on similar polymers and the results of this work are also reported. The information is of fundamental importance in order to elucidate the role of phosphoric acid in the conductivity mechanism of AB-PBI.

\section{Experimental}

Crosslinked poly(2,5-benzimidazole) (AB-PBI) membranes were obtained from FuMA-Tech $\mathrm{GmbH}$, St. Ingbert, Germany and were additionally purified by heating at $150{ }^{\circ} \mathrm{C}$ for $30 \mathrm{~min}$.

Doped membranes were prepared by immersion in an aqueous solution of ortho-phosphoric acid (PA, $85 \mathrm{wt} \%$, purchased from Merck) at $110{ }^{\circ} \mathrm{C}$ for different time periods to modulate their doping degree. The membranes were weighed before and after the immersion in acid. An AB-PBI membrane with a weight change of $42 \mathrm{wt} \%$ due to the absorption of aqueous phosphoric acid was obtained after an immersion time of $2 \mathrm{~min}$. A weight change of $185 \mathrm{wt} \%$ after $10 \mathrm{~min}$, a change of $286 \mathrm{wt} \%$ after 1 hour, and a change of $349 \mathrm{wt} \%$ after overnight immersion in aqueous acid were achieved (see also the calibration curve shown in Fig. 1).

Membrane thickness was determined using a Mahr Inductive Dial Comparator 2000 Extramess and a Planolith measuring table. The thickness ranges from $50 \mu \mathrm{m}$ for the undoped material to approximately $100 \mu \mathrm{m}$ for the highest doping levels.

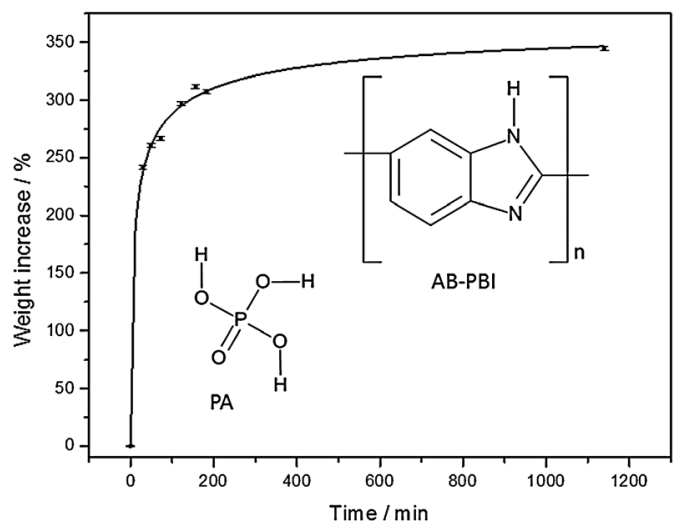

Fig. 1 Calibration curve of the doping level: phosphoric acid degree is correlated with the immersion time of the polymer membranes in acid solution. Points represent the experimental data. The molecular structure of the repeating units of poly(2,5-benzimidazole), called AB-PBI, is schematically drawn together with the ortho-phosphoric acid, called PA.
Raman measurements were performed with a Bruker RAM II FT-Raman module coupled to a VERTEX70 spectrometer. A Ge diode detector was employed. For the excitation of the Raman modes, a Nd:YAG laser with a wavelength of $\lambda=1064 \mathrm{~nm}$ was used. Excitation with shorter wavelength, i.e. starting in the visible range, was not used to avoid fluorescence phenomena, which considerably disturb the detection of Raman spectra. For the used setup, the spectral resolution was about $4 \mathrm{~cm}^{-1}$.

\section{Results and discussion}

In Fig. 2, the FT-Raman spectral features observed for pristine AB-PBI and AB-PBI doped with four different phosphoric acid concentrations are shown in the $500-2000 \mathrm{~cm}^{-1}$ wavenumber range. Three spectral regions, denoted by Greek letters, can be distinguished in the Raman spectra, delimited by dotted vertical lines.

\section{Undoped AB-PBI}

The Raman spectrum of the undoped membrane is shown in Fig. 2a. The most prominent bands are located between 1500 and $1650 \mathrm{~cm}^{-1}$ ( $\gamma$ region), the most intense of them peaking at 1546 and $1614 \mathrm{~cm}^{-1}$. All these bands can be attributed to

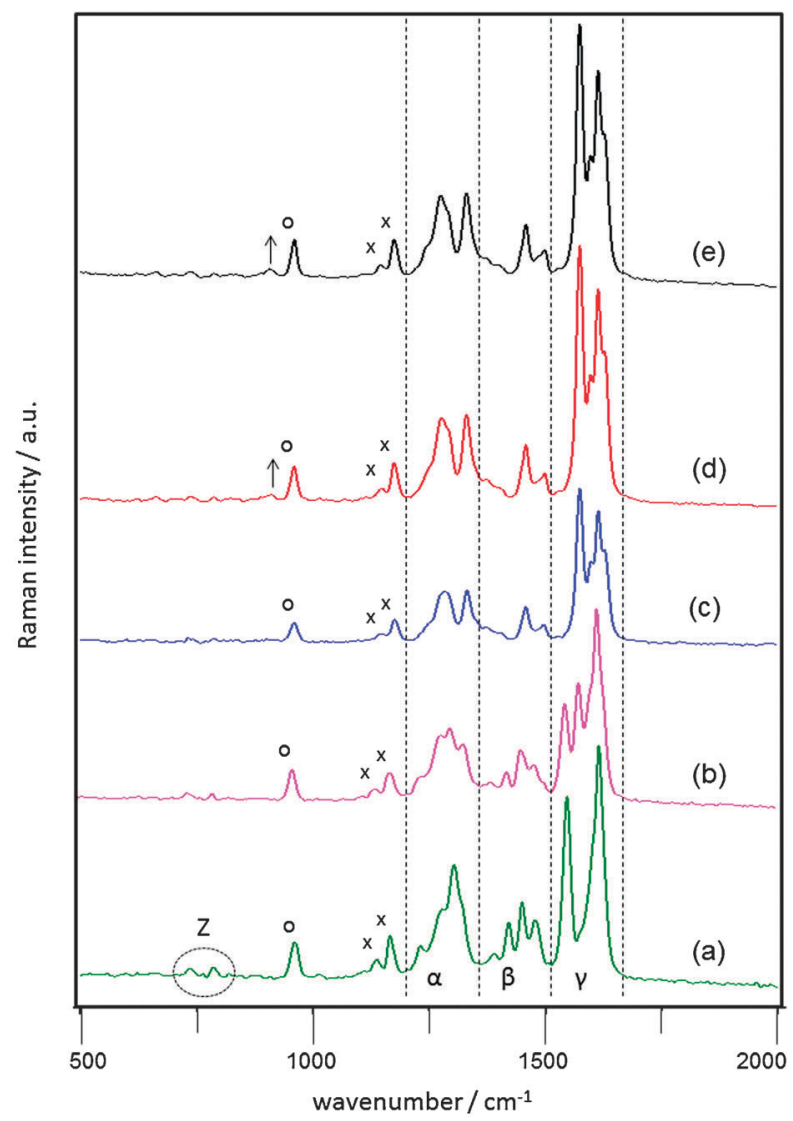

Fig. 2 FT-Raman spectra of pristine AB-PBI (a) and phosphoric acid-doped AB-PBI with four different degrees of phosphoric acid doping: $42 \mathrm{wt} \%$ (b), $185 \mathrm{wt} \%$ (c), $286 \mathrm{wt} \%$ (d), and $349 \mathrm{wt} \%$ (e). The important peaks, which are discussed in the text, are marked with symbols in the figure. The dotted lines separate three spectral regions denoted by Greek letters. Table 1 reports the attributions of the Raman signals. 
Table 1 Assignments of Raman signals of pristine AB-PBI and AB-PBI highly doped with phosphoric acid

\begin{tabular}{llll}
\hline Symbol $^{a}$ & AB-PBI & Doped AB-PBI & Assignment \\
\hline $\mathrm{Z}$ & $737(\mathrm{w}), 787(\mathrm{w})$ & - & $\mathrm{C}-\mathrm{H}$ out-of-plane ring deformation \\
$\uparrow$ & - & $964(\mathrm{w})$ & Free phosphoric acid \\
$\mathrm{O}$ & $964(\mathrm{w})$ & $1134(\mathrm{w}), 1176(\mathrm{w})$ & Ring breathing vibration of benzene ring \\
$\mathrm{x}$ & $1134(\mathrm{w}), 1176(\mathrm{w})$ & $\mathrm{C}-\mathrm{C}$ skeletal stretching \\
$\alpha$ & $1232(\mathrm{w}), 1279(\mathrm{~m}), 1305(\mathrm{~m})$ & $\mathrm{C}-\mathrm{H}$ in-plane bending vibrations \\
$\beta$ & $1390(\mathrm{w}), 1421(\mathrm{~m}), 1450(\mathrm{~m}), 1477(\mathrm{~m})$ & $1460(\mathrm{~m}), 1500(\mathrm{w})$ & $\mathrm{C}=\mathrm{C} / \mathrm{C}=\mathrm{N}$ benzimidazole ring stretching vibrations \\
$\gamma$ & $1546(\mathrm{~s}), 1614(\mathrm{~s})$ & $1574(\mathrm{~s}), 1601(\mathrm{sh}), 1614(\mathrm{~s}), 1630(\mathrm{sh})$ & $\mathrm{C}=\mathrm{C} / \mathrm{C}=\mathrm{N}$ benzimidazole ring stretching vibrations \\
${ }^{a}$ Symbols are used in Fig. $2 .{ }^{b}$ Numbers represent the Raman peak wavenumbers expressed in $\mathrm{cm}^{-1}$. Peak intensities are reported in brackets \\
\multicolumn{2}{l}{ (s, strong; m, medium; w, weak; sh, shoulder). }
\end{tabular}

the benzimidazole ring stretching vibration, $\mathrm{C}=\mathrm{C}$ and $\mathrm{C}=\mathrm{N}$ stretching, respectively. For a standard poly $\left(2,2^{\prime}-(m\right.$-phenylene)5,5'-bibenzimidazole) membrane a slight shift at higher frequencies was reported by Quartarone et al. (peaks were observed at 1539 and $\left.1600 \mathrm{~cm}^{-1}\right)^{33}$ and Sannigrahi et al. (peaks were observed at 1533,1592 , and $1610 \mathrm{~cm}^{-1}$ ). ${ }^{34,35}$

In the spectral region called $\beta$ around $1440 \mathrm{~cm}^{-1}$ the four well resolved weaker peaks at $1390,1421,1450$, and $1477 \mathrm{~cm}^{-1}$ can also be ascribed to benzimidazole ring stretching vibrations. Medium or strong intense peaks in the spectral $\alpha$ region between 1200 and $1350 \mathrm{~cm}^{-1}$ (maxima at 1232, 1279, and $1305 \mathrm{~cm}^{-1}$ ) correspond to $\mathrm{C}-\mathrm{H}$ in-plane vibrations. The two bands at 1134 and $1176 \mathrm{~cm}^{-1}$ are tentatively assigned to $\mathrm{C}-\mathrm{C}$ skeletal stretching ${ }^{33,36-38}$ (peaks marked with crosses in Fig. 2).

The band with maximum at $964 \mathrm{~cm}^{-1}$ (peak marked with a circle in Fig. 2) is most likely due to the ring breathing vibration of the benzene rings, as observed in Raman spectra of other polymers containing benzimidazole units. ${ }^{33,38,39}$ Finally, the weak and broad peaks at lower wavenumbers, around $750 \mathrm{~cm}^{-1}$, are due to $\mathrm{C}-\mathrm{H}$ out-of-plane ring deformation ${ }^{33,40}$ and are labeled with the letter $\mathrm{Z}$ in a dotted circle in Fig. 2a. The assignment of the above reported bands is schematically given in Table 1 .

\section{Phosphoric acid doped AB-PBI}

After doping with phosphoric acid a shift and change in the relative intensity are observed for many of the Raman bands, especially in the $950-1800 \mathrm{~cm}^{-1}$ spectral region. The most striking difference between the spectra of undoped and doped membranes is the lack of the bands at 1546, 1421, and around $750 \mathrm{~cm}^{-1}$ in the spectra of the doped polymers. The following discussion strengthens the attribution of chemical hydrogen bonding interactions between AB-PBI polymer chains and phosphoric acid. Moreover, it is suggested that PA acts not only as a proton donor, but also as a swelling agent. PA seems to be responsible not only for the proton conduction but also for plasticizing processes in the membranes.

The use of Raman spectroscopy as a tool to study the PBI type polymer is very rare. Recently meta-PBI, pyridine based PBI (PyPBI) random homo and copolymers and their composites have been characterized by Raman spectroscopy. ${ }^{33,34,39}$ Our results match well with the previous data. ${ }^{33,34,39}$

The vibrational assignments were made also by comparing the previously published vibrational assignments of poly-2,2'-mphenylene-5, $5^{\prime}$-bibenzimidazole (PBI4N), ${ }^{10,37,39}$ benzimidazole $^{41,42}$ and pyridine. ${ }^{43}$

Fig. 2b-e show the modifications of the Raman spectrum when prolonging the contact of the polymer membrane with phosphoric acid, i.e. when increasing the molar fraction of phosphoric acid in the membrane material. From these spectra it is evident that the addition of ortho-phosphoric acid leads to a substantial change in the optical response of the materials. The spectrum in Fig. 2e corresponds to the polymer membrane retained in a solution of $85 \%$ PA overnight.

No significant changes are observed between Fig. $2 \mathrm{~d}$ and e so that we consider that the doping degree has reached the saturation level. Our assumption is supported by the results reported in Fig. 1: the weight increase of the AB-PBI membranes doped with PA does not grow linearly but reaches a plateau when prolonging the immersion time of the membrane in phosphoric acid.

Evidence of physical-chemical interactions between polymer chains and phosphoric acid is the band shifting of the benzimidazole ring vibrations in the $1500-1650 \mathrm{~cm}^{-1}$ spectral $\gamma$ region. The new bands, either $\mathrm{C}=\mathrm{C}$ stretching $\left(1574 \mathrm{~cm}^{-1}\right)$ or $\mathrm{C}=\mathrm{N}$ stretching $\left(1601,1614\right.$, and $\left.1630 \mathrm{~cm}^{-1}\right)$, have to be associated with the formation of bonds between the acid protons of the PA and the nitrogens of the imidazole rings. In particular, as the polymer membrane is progressively doped with phosphoric acid, the prominent peak at $1546 \mathrm{~cm}^{-1}$ recorded in the Raman spectrum of pristine AB-PBI (Fig. 2a) gradually disappears: for AB-PBI at $42 \mathrm{wt} \%$ degree of PA doping, the peak intensity is already reduced to $c a$. $60 \%$ of its original value (Fig. 2b). Proportionally, a new peak at $1574 \mathrm{~cm}^{-1}$ appears until it reaches a maximum in intensity for high values of PA. The Raman mode at $1546 \mathrm{~cm}^{-1}$ is diagnostic of the interactions between the polymer and the acid and disappears when the membrane environment is strongly rich in PA. Finally, the band at higher wavelength, a peak at $1614 \mathrm{~cm}^{-1}$, does not change significantly when increasing the molar fraction of PA. These effects are compatible with the establishment of hydrogen bonds among the phosphoric acid and the polymer chains, and with the hindering of some of the polymer backbone vibrational modes due to the acid species.

To confirm the formation of hydrogen bonding networks in the phosphoric acid doped AB-PBI membranes, the band shifts in the spectral $\beta$ region around $1440 \mathrm{~cm}^{-1}$ can be also considered. The signals are attributed to benzimidazole ring stretching vibrations and the well resolved medium intense peak at $1421 \mathrm{~cm}^{-1}$ recorded in the Raman spectrum of pristine AB-PBI progressively reduces in intensity as the doping level increases: for AB-PBI at $42 \mathrm{wt} \%$ degree of PA doping, the peak intensity is already reduced to $c a .55 \%$ of its original value (Fig. 2b). For higher doping level, the Raman peak is completely absent. The two original peaks at 1450 and $1477 \mathrm{~cm}^{-1}$ (Fig. 2a) 
are shifted to higher wavenumbers $\left(1460\right.$ and $1500 \mathrm{~cm}^{-1}$ in Fig. 2e).

For $\beta$ and $\gamma$ regions, the Raman signals are mostly related to the main structure of the polymer. The acid uptake introduces modifications not only in the inter-chain dipole-dipole interactions but also in the segmental motion of the polymer backbone chains, which are strongly coupled.

Attention should be paid to the appearance of a new weak band at $911 \mathrm{~cm}^{-1}$ as the doping degree increases (the peak marked with an arrow in Fig. 2d and e). A similar signal at the same wavenumber but with a relatively higher amplitude was found in the Raman spectrum of concentrated phosphoric acid itself. $^{39}$

According to Li et al., the band is attributed to the presence of pure and free PA molecules. Thus, after a maximum degree of doping is reached, excess acid exists in the membranes. The presence of free PA at a high doping level has been observed by several authors in phosphoric acid doped PBI membranes using various techniques. ${ }^{7,9,44}$ The very low intensity of the signal shows that the large majority of PA molecules are interacting with the polymer chains, i.e. hydrogen bonds between the protonated imidazole rings in the polymer chain and the phosphoric acid molecules occur. At the high doping levels of the saturation stage, the membrane material shows a high proton conductivity and is most suitable for the use in fuel cells.

The Raman pattern of the broad band in the $1200-1350 \mathrm{~cm}^{-1}$ $\alpha$ region changes for increasing doping levels and two peaks of medium intensity at 1279 and $1333 \mathrm{~cm}^{-1}$ are distinguishable (Fig. 2c-e). The signals are due to $\mathrm{C}-\mathrm{H}$ in-plane bending vibrations and therefore are indicative of chain distortion in the polymer. In a recent paper, Di Noto et al. suggested that in undoped PBI polymers the $\mathrm{C}-\mathrm{H}$ in-plane bending modes tend to be coupled with the $\mathrm{N}-\mathrm{H}$ modes. ${ }^{10}$ In comparison to the signals of the $\alpha$ region of undoped AB-PBI, there is a shift to the higher end of the $\alpha$ wavenumber range. The Raman results reported in Fig. 2 suggest that the uptake of phosphoric acid induces a swelling of the polymer so that the polymer chains seem to be more distantly related. The $\alpha$ region can be considered to be diagnostic of the interactions of AB-PBI chains with the environment.

After doping with PA, neither shift nor strong intensity changes are observed for the band peaking at $964 \mathrm{~cm}^{-1}$ (the band marked with a circle in Fig. 2). Therefore, the influence of PA on the ring breathing vibration of the benzene rings is negligible. This observation also supports the attribution of interactions between phosphoric acid molecules and AB-PBI polymer chains due to the formation of hydrogen bonds involving only the imidazole ring. As expected, there is no noticeable influence on the vibrational breathing and stretching of the benzene rings. The free acid molecules seem to simply swell the polymer strands. The assignments of the Raman signals observed for the highly doped AB-PBI membranes are reported in Table 1 together with pristine AB-PBI to simplify a comparison.

The interactions of the AB-PBI membrane with PA can be mostly easily detected by the difference spectra reported in Fig. 3. Difference spectra are obtained by subtracting the spectrum of the pure AB-PBI membrane from the spectra of

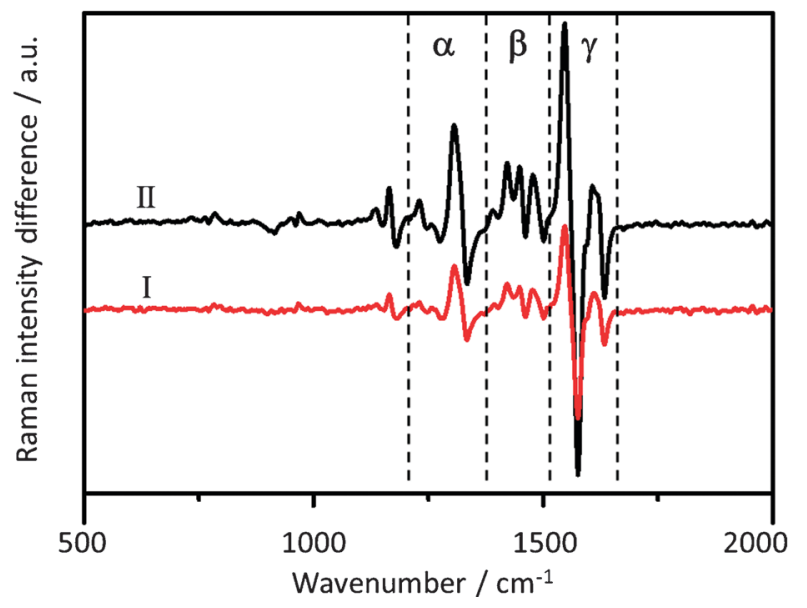

Fig. 3 Difference spectra obtained by subtracting the Raman spectrum of a pure AB-PBI membrane (Fig. 2a) from the spectrum in Fig. $2 b$ of the doped-membrane with $43 \mathrm{wt} \%$ doping degree (I) and from the spectrum in Fig. 2e of the doped membrane with $349 \mathrm{wt} \%$ doping degree (II).

the doped-membrane. In Fig. 3 are presented the two limiting cases, with lower ( $42 \mathrm{wt} \%$ ) and higher (349 wt\%) doping levels, in relation to the Raman spectrum of Fig. $2 b$ and e, respectively. The spectra were normalized with the band centred at $964 \mathrm{~cm}^{-1}$ before subtraction. Spectral profiles I and II show the presence of increasing bands in the $\gamma$ regions $(\mathrm{C}=\mathrm{C}$ and $\mathrm{C}=\mathrm{N}$ stretching $)$ due to the interaction of the acid protons of the PA with the nitrogens of the imidazole rings. In the $\beta$ regions the signals are due to the benzimidazole ring stretching vibrations so that the acid uptake introduces modifications also in the segmental motion of the polymer backbone chains. The $\alpha$ region in the difference spectra, which is due to the $\mathrm{C}-\mathrm{H}$ in plane bending vibrations, confirms the chain distortion in the polymer.

To check the stability of the doping degrees of the AB-PBI membranes doped with phosphoric acid, additional Raman measurements have been performed one month later with the same samples. In the meantime, the samples have been stored in sealed plastic bags, in darkness and at room temperature to limit interactions with atmosphere and hydration/dehydration processes. No evidence of further changes of the samples due to ageing or incomplete equilibration has been observed in the spectra: the peak due to free phosphoric acid (marked with arrows in Fig. 2) does not change in intensity and the Raman signals due to the formation of hydrogen bonds do not show significant variations. The doping and storage procedures for the membrane can be considered suitable for future tests involving phosphoric acid.

\section{Conclusions}

This paper reports a detailed study of the optical modes which are a powerful diagnostic of interactions between the chains of poly(2,5-benzimidazole) (AB-PBI) and the environment. AB-PBI membranes doped with phosphoric acid at various degrees have been prepared and investigated in regard to their spectroscopic properties.

The FT-Raman examination has provided information on the presence of hydrogen bonds between phosphoric acid and the $\mathrm{N}-\mathrm{H}$ groups of the polymer. The shifts observed in 
the Raman scattering peaks of the benzimidazole ring indicate that molecules of phosphoric acid interact with each repeat unit of AB-PBI, corresponding to the available nitrogen sites.

At high doping levels, i.e. for high molar fractions of phosphoric acid, all the nitrogen sites of AB-PBI are protonated and an additional Raman signal corresponding to free phosphoric acid molecules can be detected.

The Raman region of the $\mathrm{C}-\mathrm{H}$ vibrations provides insight into the swelling process in the polymer. It was shown by Raman measurements performed one month later that the samples have reached their equilibrium state using the described doping procedure.

This work demonstrates the Raman approach to improve the knowledge on acid doped AB-PBI: phosphoric acid seems to be responsible not only for the proton conduction necessary for the use in fuel cells, but also for plasticizing processes which swell the benzimidazole polymer membranes. A detailed assignment of the Raman modes can be also useful for further in situ/in operandum investigation on HT-PEFC.

\section{Acknowledgements}

We thank our colleagues from Forschungszentrum Jülich, Jennifer Bachhausen ( $\mathrm{ZCH}$ ) for helping us with the FT-Raman experiments, and Fang Liu (IEK-3) for the contribution to the sample preparation. FC is grateful to the University of Padova for providing the opportunity to spend a research year in Forschungszentrum Jülich.

\section{References}

1 R. Borup, J. Meyers, B. Pivovar, Y. S. Kim, R. Mukundan, N. Garland, D. Myers, M. Wilson, F. Garzon, D. Wood, P. Zelenay, K. More, K. Stroh, T. Zawodzinski, J. Boncella, J. E. McGrath, M. Inaba, K. Miyatake, M. Hori, K. Ota, Z. Ogumi, S. Miyata, A. Nishikata, Z. Siroma, Y. Uchimoto, K. Yasuda, K. I. Kimijima and N. Iwashita, Chem. Rev., 2007, 107, 3904-3951.

2 F. Conti, E. Negro, V. Di Noto, G. Elger, T. Berthold and S. Weber, Int. J. Hydrogen Energy, 2012, 37, 6317-6325.

3 J. N. Asensio, E. M. Sánchez and P. Gómez-Romero, Chem. Soc. Rev., 2010, 39, 3210-3239.

4 T. Smolinka, S. Rau and C. Hebling, in Hydrogen and Fuel Cells, ed. D. Stolten, Wiley-VCH, Weinheim, 2010, p. 271.

5 S. Bose, T. Kuila, T. X. H. Nguyen, N. H. Kim, K. Lau and J. H. Lee, Prog. Polym. Sci., 2011, 36, 813-843.

6 J. T. Wang, R. F. Savinell, J. S. Wainright, M. Litt and H. Yu, Electrochim. Acta, 1996, 41, 193-197.

7 Q. Li, J. O. Jensen, R. F. Savinell and N. J. Bjerrum, Prog. Polym. Sci., 2009, 34, 449-477.

8 J. Hu, J. Luo, P. Wagner, O. Conrad and C. Agert, Electrochem. Commun., 2009, 11, 2324-2327.

9 F. J. Nores-Pondal, M. P. Buera and H. R. Corti, J. Power Sources, 2010, 195, 6389-6397.

10 V. Di Noto, M. Piga, G. A. Giffin, E. Quartarone, P. Righetti, P. Mustarelli and A. Magistris, Phys. Chem. Chem. Phys., 2011, 13, 12146-12154.

11 E. W. Choe, J. Appl. Polym. Sci., 1994, 53, 497-506.

12 J. S. Wainright, J. T. Wang, D. Weng, R. F. Savinell and M. Litt, J. Electrochem. Soc., 1995, 142, L121-L123.

13 J. S. Wainright, M. H. Litt and R. F. Savinell, in Handbook of Fuel Cells Fundamentals, Technology and Applications, ed. W. Vielstich,
A. Lamm and H. A. Gasteiger, John Wiley \& Sons Ltd, Chichester, 2003, vol. 3, pp. 436- 446.

14 Y. Iwakura, Y. Imai and K. Uno, J. Polym. Sci., Part A: Gen. Pap., 1964, 2, 2605-2615.

15 H. J. Kim, S. Y. Cho, S. J. An, J. Y. Kim, Y. C. Eun, J. Y. Kim, H. K. Yoom, H. J. Kweon and K. H. Yew, Macromol. Rapid Commun., 2004, 25, 894-897.

16 J. Jouanneau, R. Mercier, L. Gonon and G. Gebel, Macromolecules, 2007, 40, 983-990.

17 L. Xiao, H. Zhang, T. Jana, E. Scanlon, R. Chen, E. W. Choe, L. S. Ramanathan, S. Yu and B. C. Benicewicz, Fuel Cells, 2005, 8, 287-295.

18 A. Carollo, E. Quartarone, C. Tomasi, P. Mustarelli, F. Belotti, A. Magistris, F. Maestroni, M. Parachini, L. Garlaschelli and P. P. Righetti, J. Power Sources, 2006, 160, 175-180.

19 Q. Li and J. O. Jensen, in Membranes for Energy Conversion 2, ed. K. V. Peinenmann and S. Pereira Nunes, Wiley-VCH, Weinheim, 2008, pp. 61-96.

20 S. Bhadra, N. H. Kim, J. S. Choi, K. Y. Rhee and J. H. Lee, J. Power Sources, 2010, 195, 2470-2477.

21 J. Lobato, P. Canizares, M. A. Rodrigo, J. J. Linares and G. Manjavacas, J. Membr. Sci., 2006, 280, 351-362.

22 Q. Li, R. He, J. O. Jensen and N. J. Bierrum, Fuel Cells, 2004, 4, $147-159$.

23 Q. Li, R. He, J. A. Gao, J. O. Jensen and N. J. Bierrum, J. Electrochem. Soc., 2003, 150, A1599-A1605.

24 R. He, Q. Li, A. Bach, J. O. Jensen and N. J. Bjerrum, J. Membr. Sci., 2006, 277, 38-45.

25 J. N. Asensio, S. Borrós and P. Gómez-Romero, Electrochem. Commun., 2003, 5, 967-972.

26 H.-J. Kim, S. Y. Cho, S. J. An, Y. C. Eun, J.-Y. Kim, H.-K. Yoon, H.-J. Kweon and K. H. Yew, Macromol. Rapid Commun., 2004, 25, 894-897.

27 J. J. Linares, C. Sánches, V. A. Paganin and E. R. González, ECS Trans., 2011, 41, 1579-1593.

28 C. Wannek, B. Kohnen, H.-F. Oetjen, H. Lippert and J. Mergel, Fuel Cells, 2008, 8, 87-95.

29 C. Wannek, W. Lehnert and J. Mergel, J. Power Sources, 2009, 192, 258-266.

30 K. Wippermann, C. Wannek, H.-F. Oetjen, J. Mergel and W. Lehnert, J. Power Sources, 2010, 195, 2806-2809.

31 J. A. Asensio and P. Gómez-Romero, Fuel Cells, 2005, 5, 336-343.

32 R. M. Silverstein and F. X. Webster, Spectroscopic identification of organic compounds, John Wiley \& Sons Inc., NY, 2002.

33 E. Quartarone, A. Magistris, P. Mustarelli, S. Grandi, A. Carollo, G. Z. Zukowska, J. E. Garbarczyk, J. L. Nowinski, C. Gerbaldi and S. Bodoardo, Fuel Cells, 2009, 9(4), 349-355.

34 A. Sannigrahi, S. Ghosh, S. Maity and T. Jana, Polymer, 2010, 51 5929-5941.

35 A. Sannigrahi, S. Ghosh, S. Maity and T. Jana, Polymer, 2011, 52, 4319-4330.

36 P. Musto, F. E. Karasz and W. J. MacKnight, Polymer, 1989, 30, 1012.

37 P. Musto, F. E. Karasz and W. J. MacKnight, Polymer, 1993, 34, 2934-2945.

38 V. Deimede, G. A. Voyiatzis, J. K. Kallitsis, L. Qingfeng and N. J. Bjerrum, Macromolecules, 2000, 33, 7609-7617.

39 Q. Li, R. He, R. W. Berg, H. A. Hjuler and N. J. Bjerrum, Solid State Ionics, 2004, 168, 177-185.

40 G. Xue, J. Dong and J. F. Zhang, Macromolecules, 1991, 24, 4195-4198.

41 R. Infante-Castillo, L. A. Rivera-Montalvo and S. P. HernandezRivera, J. Mol. Struct., 2008, 877, 10-19.

42 M. A. Morsy, M. A. Al-Khaldi and A. Suwaiyan, J. Phys. Chem. A, 2002, 106, 9196-9203.

43 F. P. Urena, M. F. Gomez, J. J. L. Gonzalez and E. M. Torres, Spectrochim. Acta, Part A, 2003, 59, 2815-2839.

44 Y.-L. Ma, J. S. Wainright, M. H. Litt and R. F. Savinell, J. Electrochem. Soc., 2004, 151, A8-A16. 